\title{
Non-Alcoholic Fatty Liver Disease: Cause or Effect of Metabolic Syndrome
}

\author{
Christoph Grander Felix Grabherr Alexander R. Moschen Herbert Tilg \\ Department of Internal Medicine I, Gastroenterology, Endocrinology \& Metabolism, Medical University Innsbruck, Innsbruck, Austria
}

\section{Keywords}

Adiponectin · Cytokines · Inflammation .

Insulin resistance . Treatment

\section{Summary}

Non-alcoholic fatty liver disease (NAFLD) is the most common liver disease throughout the world. Pathophysiological insights into this disease have recently illustrated that various factors such as insulin resistance, innate immunity, metabolic inflammation, and the microbiota are of relevance. NAFLD, metabolic syndrome (MS), and type 2 diabetes (T2D) share many pathophysiological aspects, and inflammatory processes in the adipose tissue, gut, and liver have evolved to be of exceptional importance. Most of NAFLD patients are obese and encounter a high risk of developing MS and T2D. NAFLD, however, is also highly common in subjects with MS and T2D. Furthermore, reflecting its nature of a multisystem disease, NAFLD is associated with a high prevalence and incidence of cardiovascular and chronic kidney disease. These facts require screening strategies for MS/ T2D in NAFLD patients and vice versa. Thus, the question of cause or effect cannot be answered as MS and NAFLD share many pathomechanisms, and at the time of either diagnosis both frequently coexist. This is also reflected by a global prevalence rate of $25 \%$ for both NAFLD and MS. For this reason, it is crucial that physicians are aware of the 'unholy liaison' between MS, T2D, and NAFLD.

(C) 2016 S. Karger GmbH, Freiburg

\section{Introduction}

Non-alcoholic fatty liver disease (NAFLD) has become the most common liver disease in the world mainly because of the obesity pandemic [1]. It is assumed that the global prevalence of this disorder is similar to metabolic syndrome (MS) with around $25 \%$. In $80 \%$ of affected subjects NAFLD is associated with obesity, although the disease may also develop in lean individuals. NAFLD may either present as simple steatosis (non-alcoholic fatty liver (NAFL)) or evolve in 10-20\% towards its inflammatory complication, i.e. non-alcoholic steatoheptatitis (NASH). Although the overall rate of NASH in the NAFLD population is unclear, certain studies propose that NASH may affect up to $5 \%$ of the general population. The disease further progresses towards liver cirrhosis and hepatocellular carcinoma, a complication which is increasingly observed also in the non-cirrhotic NAFLD population $[2,3]$. The high rate of hepatocellular carcinoma in NAFLD requires special attention.

In the last decade, insulin resistance has been considered as the major underlying pathophysiological mechanism although it is not present in all affected individuals. Especially obesity-induced insulin resistance is a dominant pathophysiological factor underlying NAFLD. Inflammatory aspects are also of crucial importance particularly in NASH and NASH-associated fibrosis, which commonly develops subsequent to overwhelming inflammatory events in the liver [4]. Chronic inflammation characterizes many metabolic disorders such as obesity, MS, type 2 diabetes (T2D), or NAFLD. This so-called low-grade inflammation has also been termed metabolic inflammation as it has been initially observed in obesity and T2D $[5,6]$. It is currently believed that, besides lipotoxic mechanisms, inflammatory pathways contribute substantially to insulin resistance observed in NAFLD.

Whereas NAFLD is commonly associated with insulin resistance and MS, the presence of MS or T2D should encourage every physician to search for accompanying NAFLD as all these disor-

\section{KARGER}

() 2016 S. Karger GmbH, Freiburg
Univ.-Prof. Dr. Herbert Tilg

Universitätsklinik für Innere Medizin I - Endokrinologie, Gastroenterologie und Stoffwechselerkrankungen Medizinische Universität Innsbruck

Anichstraße 35, 6020 Innsbruck, Austria

herbert.tilg@i-med.ac.at 
ders frequently coexist. This immediately raises the question of what comes first. This article will discuss current pathways in the etiology of NAFLD and present some clinical aspects.

\section{Definition of NAFLD}

Liver steatosis is commonly diagnosed by ultrasonography, which is an acceptable screening tool to suggest the presence of NAFLD. A more accurate diagnosis of steatosis is possible when using proton magnetic resonance spectroscopy, which is, however, not readily available. NASH with features of inflammation, ballooning, and fibrosis can only be diagnosed by liver biopsy. Fibrosis has been demonstrated to reflect the most important prognostic factor as the degree of fibrosis directly correlates with the prognosis of liver disease $[7,8]$. Unfortunately, definite diagnosis of the presence of fibrosis and NASH can only be made by liver histology which is rarely performed in NAFLD patients except in selected situations and/or clinical trials [9-13]. Besides ultrasonography, the use of transient elastography is recommended to discover advanced fibrosis; however, in the case of severe obesity this method has certain shortcomings [14]. Although there might exist a typical profile of elevated liver enzyme values with increased serum alanine aminotransferase (ALT) and/or $\gamma$-glutamyl transferase (GGT) levels, many NAFLD patients exhibit normal values.

\section{Pathophysiological Aspects: Regulation of Insulin Resistance}

\section{Hepatic Steatosis and Insulin Resistance}

NASH patients commonly present with metabolic inflammation which is mirrored by elevated levels of high-sensitivity C-reactive protein, ferritin, or interleukin (IL)-1 receptor antagonist serum levels. It is currently not understood why certain NAFLD patients exhibit an inflammatory phenotype and progress towards fibrosis and cirrhosis while most do not. Evidence is increasing that systemic, i.e. metabolic inflammation, besides being prognostically highly relevant, is also a driver of hepatic insulin sensitivity. Research from the last decade has clearly shown that NAFLD is a multisystem disorder which is caused by multiple parallel hits [15]. It is currently believed that besides lipotoxicity, insulin resistance, innate immunity, mitochondrial dysfunction, endoplasmic reticulum stress, and the intestinal microbiota are involved in the disease process.

Insulin acts in all cells by means of binding to its specific receptor and activating a cascade of intracellular signaling events. After binding, the insulin receptor phosphorylates itself as well as several members of the insulin receptor substrate family. The primary pathophysiological mechanisms of insulin resistance induced by inflammatory mediators are very likely due to interference at the signaling level [16]. Proinflammatory cytokines and transcription factors are highly expressed in the adipose tissue or liver in case of obesity, and different immune cells and tissue macrophages con- tribute to metabolic inflammation and associated insulin resistance. Besides inflammation, lipid metabolism abnormalities have been demonstrated in insulin-resistant states, and certain lipids such as free fatty acids might interfere with insulin signaling, thereby contributing to insulin resistance $[17,18]$. The gut microbiota has evolved as another key player in insulin resistance, as recognized recently [19]. Importantly, hepatic steatosis is not unambiguously associated with insulin resistance, and situations might exist where hepatic steatosis is associated even with increased insulin sensitivity [20].

\section{Inflammation: The Key Driving Force in NASH}

Fibrosis is commonly considered the net end result of overwhelming and uncontrolled chronic inflammation. This is also the case in NASH, although it has been speculated that fibrosis might also evolve independent from inflammation in a small number of subjects. Importantly, even simple fatty liver may progress towards NASH and fibrosis [21], suggesting that either intermittent inflammation exists and/or the disease itself exhibits an undulating course. This is difficult to prove in humans because of the shortcomings of noninvasive diagnostic possibilities. Nevertheless, the importance of proinflammatory cytokines in NASH has been repeatedly demonstrated.

The liver plays a fundamental role in insulin resistance and T2D. Various inflammatory pathways involving proinflammatory cytokines such as TNF- $\alpha$ or IL- 6 are activated in the liver in states of insulin resistance $[22,23]$. Activation of the transcription factor nuclear factor-kappa B (NF- $\mathrm{BB})$ and its associated inflammatory signaling pathways are involved in hepatic insulin resistance. The IKB kinase (IKK) complex plays a crucial role in the activation of NF- $\kappa B$ by triggering phosphorylation and degradation of the inhibitory molecule IKB $\alpha$. Two studies have shown a role for IKK $\beta$ expression in the liver and in insulin resistance $[24,25]$. Cai et al. [24] generated chronic, NF- $\kappa \mathrm{B}$-driven hepatic inflammation in mice constitutively expressing low levels of IKK $\beta$ in the liver. These mice exhibited a T2D-like disease with moderate systemic insulin resistance. Arkan et al. [25] investigated mice lacking IKK $\beta$ either in hepatocytes or the myeloid compartment. Mice with liver-specific deletion of IKK $\beta$ retained their liver insulin responsiveness after consumption of a high-fat diet but developed insulin resistance in muscle and fat. Overall, elevated NF- $\kappa \mathrm{B}$ activity in the hepatocyte is associated with insulin resistance, suggesting a key importance of the hepatocyte in the regulation of hepatic insulin sensitivity. We have shown that receptor activator of NF- $\kappa B$ (RANKL), a prototypic activator of NF- $\mathrm{BB}$, also regulates hepatic insulin sensitivity [26]. All these studies clearly underline the importance of inflammatory pathways in NASH and suggest that targeting these cascades at various steps might constitute an attractive treatment approach (fig. 1).

\section{Adiponectin: The Key Adipocytokine Controlling Metabolic Processes}

Adiponectin is a protoyptic anti-inflammatory adipocytokine mainly produced by the adipose tissue [27]. It suppresses inflam- 
Fig. 1. Potentially involved hepatic pathways in metabolic inflammation and insulin resistance. (1) Interaction of insulin with its receptor results in tyrosine phosphorylation of the IRS and activation of downstream effector mechanisms such as the PI3K/AKT, the PI3K/ $\mathrm{PKC}$, and ERK pathways (not shown). Various inflammatory networks interfere with insulin signaling. Insulin signaling is attenuated either by inhibitory serine phosphorylation of the IRS or its proteasomal degradation. (2) Engagement of TNFR 1 by TNF- $\alpha$ results in activation of IKK $\beta$ via TRAF2. Besides IKK $\beta$ 's outstanding role as activator of NF-kB, the prototypic proinflammatory transcription factor, it has proven a potent catalyst of insulin resistance. (3) The TNF receptor superfamily member RANK

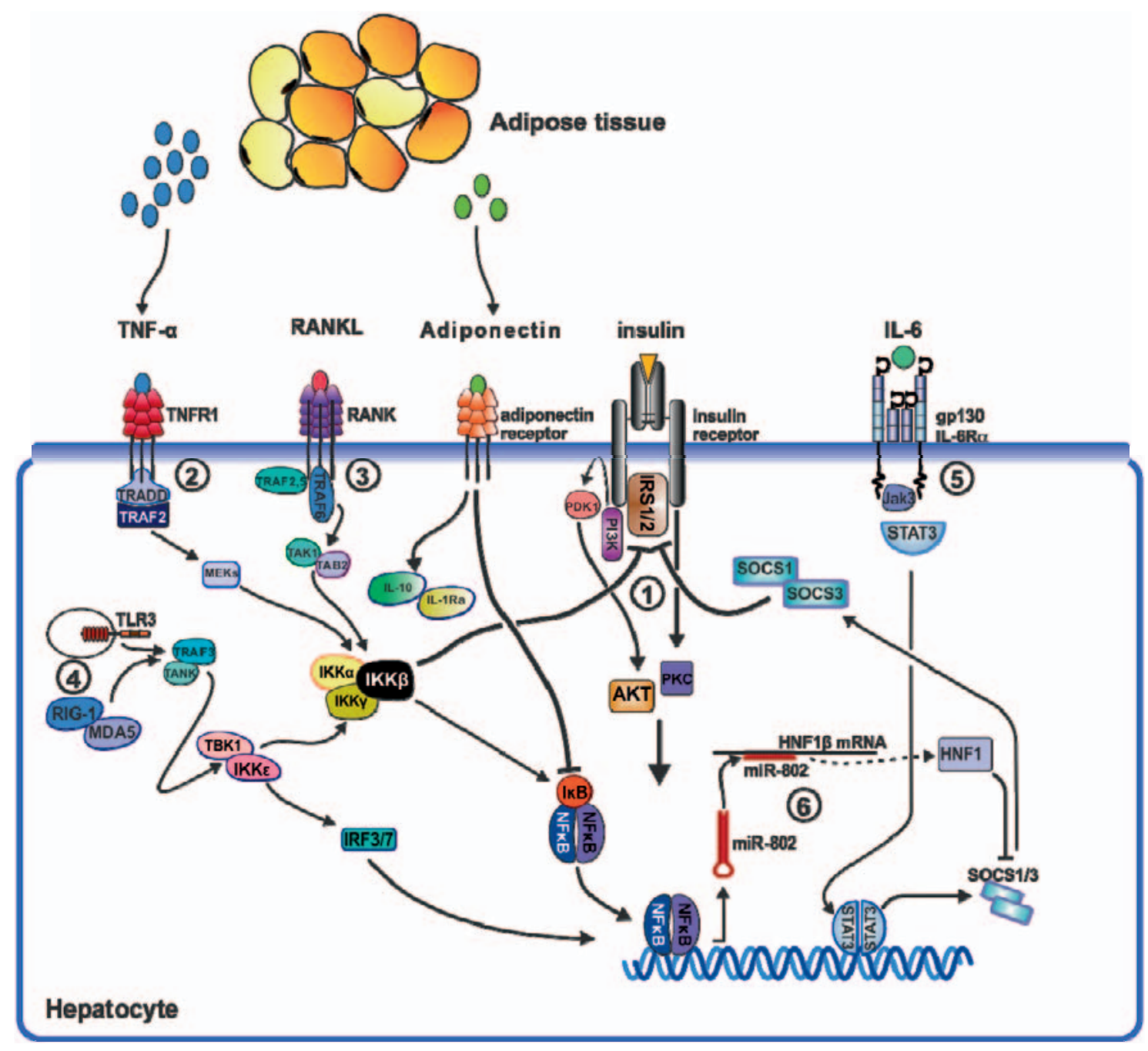
and its ligand RANKL

activate IKK $\beta$ mainly via TRAF6 and promote hepatic insulin resistance. (4) Alternatively, IKK $\beta$ activation is initiated by IKKe, a non-canonical NF- $\mathrm{KB}$ activator. (5) The obese adipose tissue produces vast amounts of IL-6 which arrives at the liver through the circulation. Activation of the IL-6Ra/gp130 receptor complex results in activation of STAT3, a potent inducer of SOCS molecules. SOCS1 and SOCS3 have been shown to impair insulin signaling by mediating ubiquitin-dependent degradation of the insulin receptor substrate. (6) Expression of SOCS1 and SOCS3 is repressed by HNF1 $\beta$, a member of the homeodomain-containing superfamily of transcription factors. Translation of HNF1 $\beta$ mRNA is post-transcriptionally regulated by miR-802, a micro RNA that is strongly upregulated in obesity. In a sense, obesity affects SOCS1 and SOCS3 production from two sides, firstly by inducing its expression via IL-6/STAT3 and secondly by suppressing the translation of its inhibitor HNF1 $\beta$ via miR-802. (7) Adipose tissue secretes adiponectin, which exhibits anti-inflammatory properties by inducing IL-10 and IL-1Ra expression. In states of obesity and insulin resistance, however, adipose tissue releases less amounts of adiponectin. Abbreviations: AKT = Protein kinase b; ERK = extracellular signal-regulated kinase; gp130 = interleukin 6 signal transducer; HNF-1 $\beta=$ hepatic nuclear factor 1 beta; IKK $\beta=$ inhibitor of kappa light chain gene enhancer in $B$ cell beta kinase; IL-1 = interleukin 1; IL-1Ra = interleukin 1 receptor antagonist; IL-6 = interleukin 6; IL-10 = interleukin 10; IRF-3/7 = interferon regulatory factor 3/7; IRS-1/2 = insulin receptor substrate 1/2; JAK-3 = janus kinase 3; MEK = mitogen-activated protein kinase; MDA-5 = melanoma differentiation-associated gene 5; miR-802 = micro RNA 802; NF- $\mathrm{kB}$ = nuclear factor kappa B; RANK = receptor activator of NF kappa B; RANKL = receptor activator of NF kappa B ligand; RIG-1 = retinoic acid-induced gene 1 ; SOCS-1/3 = suppressor of cytokine signaling $1 / 3$; STAT-3 = signal transducer and activator of transcription 3; TAB-2 = TAK1-binding protein 2 ; TAK-1 $=$ transforming growth factor beta-activated kinase 1 ; TBK-1 $=$ TANK-binding kinase 1 ; TLR-3 $=$ toll-like receptor 3 ; TNF- $\alpha=$ tumor necrosis factor alpha; TNFR-1 = tumor necrosis factor receptor 1 ;RADD $=$ tumor necrosis factor receptor 1 -associated death domain protein; TRAF-2/6 $=$ TNF receptor-associated factor 2/6; PDK1 = pyruvate dehydrogenase kinase isoenzyme 1; PI3K = phosphatidylinositol 3-kinase; PKC $\lambda$ = protein kinase $\mathrm{C}$ gamma

mation in endothelial cells via regulation of proinflammatory adhesion molecules and in many other models [28]. Adiponectin also inhibits endothelial NF- $\mathrm{BB}$ signaling and controls macrophage function [29]. Adiponectin KO mice exhibit evidence of increased local and systemic TNF- $\alpha$ production [30]. Anti-inflammatory mechanisms of adiponectin also involve the induction of other anti-inflammatory cytokines such as IL-10 and IL-1 receptor an- tagonists [31]. Another anti-inflammatory pathway controlled by adiponectin involves regulation of heme oxygenase-1 [32]. Weight loss is a very potent inducer of adiponectin synthesis [23, 33]. Plasma levels of adiponectin are markedly diminished in visceral obesity and in states of insulin resistance such as NASH, atherosclerosis, and T2D [34]. 
Adiponectin exerts anti-inflammatory effects in various animal models of liver inflammation. We have observed that the protective effect of adiponectin in concanavalin A-induced hepatotoxicity is mainly mediated by induction of IL-10, suggesting that adiponectin-induced regulation of various anti-inflammatory mechanisms throughout the body contributes to its beneficial metabolic functions [35]. Overall, evidence is overwhelming to suggest that adiponectin reflects a master adipocytokine in health and that dysregulation drives metabolic inflammation, as observed in NAFLD, MS, and T2D.

\section{Clinical Aspects: NAFLD, Metabolic Syndrome and Type 2 Diabetes - a Cluster of a Single Disease?}

\section{NAFLD: Commonly Associated with Insulin Resistance}

Obesity and related insulin resistance is the major risk factor for NAFLD. A landmark observation by Marchesini et al. [36] defined this association for the first time, describing the presence of insulin resistance in a high percentage of NAFLD subjects. Numerous reports have proven this association, and it is currently believed that more than $80 \%$ of subjects with NAFLD including lean patients exhibit insulin resistance. Insulin resistance involves various tissues, including liver, adipose and muscle tissue. It is commonly defined in non-diabetic NAFLD subjects by the product of fasting glucose (in $\mathrm{mmol} / \mathrm{l}$ ) and insulin (in $\mathrm{mU} / \mathrm{ml}$ ), divided by 22.5 (homeostatic model assessment of insulin resistance (HOMA-IR)), and reflects an accepted parameter for insulin resistance [37]. During follow-up of NAFLD patients, HOMA-IR and worsening of metabolic risk factors might identify patient groups at risk for NASH or fibrosis progression in selected cases [9-13]. Another clinically relevant aspect is the proper interpretation of elevated liver function tests. Several large studies have convincingly shown that elevated serum ALT and/ or GGT levels are associated with an increased incidence of T2D even after adjustment for several risk factors $[38,39]$. Ultrasonography-defined NAFLD exhibits a more than 2- to 5-fold risk of developing T2D over time [40]. This underlines a role for clinical surveillance of patients with 'only' liver steatosis because of their high likelihood of developing MS and/or T2D. Furthermore, each patient with liver steatosis needs a careful investigation to rule out MS/T2D either by fasting or random blood glucose or HbAlc, or standardized 75-g oral glucose tolerance testing. However, some genetic forms of NAFLD with mutations in PNPLA3 and TM6SF2 are not associated with insulin resistance or features of MS or an increased risk of T2D [41]. It has been postulated by some authors that this group accounts for potentially $20-30 \%$ of the NAFLD population.

\section{Metabolic Syndrome: Commonly Associated with NAFLD}

Subjects with MS with additional insulin resistance exhibit increased liver fat and frequently elevated ALT levels [42]. NAFLD is highly prevalent in patients with MS, and indeed, all components of MS correlate with the amount of liver steatosis. This results in the important clinical recommendation to evaluate the risk of NAFLD and vice versa the presence of NAFLD in all subjects with any component of MS. Although it is well accepted, as stated before, that the degree of fibrosis is the key prognostic factor, earlier investigations have demonstrated by using follow-up liver biopsies that a substantial proportion of patients with NAFL progress towards NASH and fibrosis, especially if metabolic risk factors worsen and T2D develops [43].

\section{T2D: Search for NAFLD}

T2D patients commonly exhibit increased liver enzymes and show a rate of NAFLD of up to $50-70 \%$, as assessed by ultrasonography [44-46]. Magnetic resonance elastography demonstrated high rates of both NAFLD and advanced fibrosis [47]. NAFLD in T2D might exert a much higher incidence of NASH compared to non-diabetic NAFLD populations, and rates between 20 and 50\% have been reported $[48,49]$. Furthermore, other prognostically relevant features such as fibrosis are much more prevalent [50]. It has also been shown that the presence of T2D in NAFLD patients is an independent predictor of moderate-to-severe fibrosis [51]. Therefore, it is suggested that every T2D should be screened for NAFLD by at least liver chemistry (although $50 \%$ of patients will exhibit normal ALT levels) and ultrasonography.

\section{NAFLD: A Multisystem Disease}

Importantly, we have learned in the last decade that NAFLD is a mirror of many potential entities and can be considered more as a 'symptom' of a complex disease. In line with this, data have accumulated that NAFLD is not only associated with a significantly increased risk of T2D but also with an increased prevalence and incidence of cardiovascular and chronic kidney disease [52]. NAFLD per se may also play a role in the pathogenesis of these extrahepatic chronic complications. This clearly implies that NAFLD patients need counseling regarding these associations to decrease the risk for cardiovascular and kidney complications.

\section{Treatment of NASH: Only Effective If Interfering with Insulin Resistance?}

NAFLD and T2D share some common pathophysiological features; therefore, it is not surprising that several therapies used in the treatment of T2D also show efficacy in the treatment of NAFLD. Although several treatments have so far been studied in NAFLD, especially targeting insulin resistance such as thiazolidinediones [53], still no medical therapy is currently approved for this disorder. Although thiazolidinediones improve insulin resistance, they result in weight gain and, importantly, were not able to reduce liver fibrosis in clinical studies [54]. The increase in adiponectin secretion and the decrease in free fatty acid release likely underlie the beneficial effect of peroxisome proliferator-activated receptorgamma (PPAR $\gamma$ ) agonists on NAFLD. Independent of the efficacy of thiazolidinediones in liver disease, several other aspects require attention. Fluid retention with the risk of congestive heart failure and also atypical bone fractures in women have been reported. Vitamin $\mathrm{E}$ also demonstrated some efficacy in large clinical trials, in- 
cluding histological improvements, but again could not improve liver fibrosis [53]. Importantly, vitamin $\mathrm{E}$ is a prototypic example of a therapeutic with a certain efficacy in NASH without affecting insulin resistance. Incretin mimetics, such as glucagon-like peptide-1 agonists, primarily stimulate glucose-dependent insulin secretion. They lead to reductions in body weight, insulin resistance, and liver transaminases. In a recently reported pilot trial, subcutaneous administration of liraglutide $(1.8 \mathrm{mg} /$ day $)$ resulted in significant decreases in liver fat content and histological resolution of NASH [55]. Bile acids have recently been shown to control metabolic and immune functions, signaling through two major receptor pathways: farnesoid X receptor (FXR), a member of the nuclear hormone receptor superfamily, and TGR5, a G protein-coupled bile acid receptor [56]. A large placebo-controlled randomized study suggested that obeticholic acid, an FXR agonist, shows promising effects in NASH with histological improvement of steatosis, lobular inflammation, and especially fibrosis [57]. One of the caveats regarding this potential therapy, however, remains the fact that it caused itching in a significant number of patients and worsened the lipid profile. Another recent approach focused on targeting PPAR $\alpha / \delta$. Here, Ratziu et al. [58] showed that elafibranor induces resolution of NASH without worsening fibrosis. Overall, all these studies support the notion that not only most therapies used to treat NASH come from the diabetes field but also that, more importantly, targeting insulin resistance and inflammation remains the cornerstone of therapeutic avenues. However, a detailed discussion of this aspect is beyond the scope of this article.

\section{Conclusion}

In this review article, we presented evidence that NAFLD, MS, and $\mathrm{T} 2 \mathrm{D}$ are highly interconnected clinical entities. This results in the very important clinical consequence that the presence of either entity should immediately result in searching for the other. The question of 'What comes first?' is difficult to answer as we have learned that (i) NAFLD is frequently accompanied by insulin resistance, (ii) diagnosis of NAFLD is commonly followed by later diagnosis of MS/T2D, and (iii) MS/T2D is extremely frequently associated with NAFLD. Therefore, we can conclude that these three entities are part of a syndrome sharing mechanisms and pathophysiology. We need to educate practitioners, internists, diabetologists, and hepatologists to share this knowledge and to look at NAFLD what it is: a complex metabolic, multisystem disease.

\section{Highlights}

- NAFLD is the most common liver disease worldwide; $10-20 \%$ of patients exhibit NASH.

- Degree of fibrosis is the most relevant prognostic marker.

- Inflammation, lipotoxicity, insulin resistance, and gut dysbiosis reflect hallmarks of NAFLD.

- NAFLD, MB, and T2D are part of a syndrome sharing pathomechanisms and treatment targets.

- Recent studies suggest that thiazolidinediones, vitamin E, obeticholic acid, and elafibrinor demonstrate some efficacy in $\mathrm{NASH}$, although they are currently not approved for this indication.

\section{Disclosure Statement}

No conflict of interest exists.

\section{References}

1 Rinella ME: Nonalcoholic fatty liver disease: a systematic review. JAMA 2015;313:2263-2273

2 Wree A, Broderick L, Canbay A, Hoffman HM, Feld stein AE: From NAFLD to NASH to cirrhosis - new insights into disease mechanisms. Nat Rev Gastroenterol Hepatol 2013;10:627-636

3 Michelotti GA, Machado MV, Diehl AM: NAFLD, NASH and liver cancer. Nat Rev Gastroenterol Hepatol 2013;10:656-665.

4 Tilg H, Hotamisligil GS: Nonalcoholic fatty liver disease: cytokine-adipokine interplay and regulation of insulin resistance. Gastroenterology 2006;131:934-945.

5 Gregor MF, Hotamisligil GS: Inflammatory mechanisms in obesity. Annu Rev Immunol 2011;29:415445.

6 Odegaard JI, Chawla A: Pleiotropic actions of insulin resistance and inflammation in metabolic homeostasis. Science 2013;339:172-177.
Angulo P, Kleiner DE, Dam-Larsen S, Adams LA, Bjornsson ES, Charatcharoenwitthaya P, Mills PR, Keach JC, Lafferty HD, Stahler A, Haflidadottir S, Bendtsen F: Liver fibrosis, but no other histologic features, is associated with long-term outcomes of patients with nonalcoholic fatty liver disease. Gastroenterology 2015;149:389-397.e310.

8 Ekstedt M, Hagstrom H, Nasr P, Fredrikson M, Stal P, Kechagias S, Hultcrantz R: Fibrosis stage is the strongest predictor for disease-specific mortality in NAFLD after up to 33 years of follow-up. Hepatology 2015;61: $1547-1554$.

9 EASL-EASD-EASO: Clinical practice guidelines for the management of non-alcoholic fatty liver disease. J Hepatol 2016;64:1388-1402.

10 EASL-EASD-EASO: Clinical practice guidelines for the management of non-alcoholic fatty liver disease. Diabetologia 2016;59:1121-1140.
Bugianesi E: EASL-EASD-EASO clinical practice guidelines for the management of non-alcoholic fatty liver disease: disease mongering or call to action? Diabetologia 2016;59:1145-1147.

12 Byrne CD, Targher G: EASL-EASD-EASO clinical practice guidelines for the management of non-alcoholic fatty liver disease: is universal screening appropriate? Diabetologia 2016;59:1141-1144.

13 Toplak H, Stauber R, Sourij H: EASL-EASD-EASO clinical practice guidelines for the management of non-alcoholic fatty liver disease: guidelines, clinical reality and health economic aspects. Diabetologia 2016; 59:1148-1149.

14 Castera L: Noninvasive evaluation of nonalcoholic fatty liver disease. Sem Liver Dis 2015;35:291-303.

15 Tilg H, Moschen AR: Evolution of inflammation in nonalcoholic fatty liver disease: the multiple parallel hits hypothesis. Hepatology 2010;52:1836-1846.

16 Samuel VT, Shulman GI: Mechanisms for insulin resistance: common threads and missing links. Cell 2012; 148:852-871. 
17 Glass CK, Olefsky JM: Inflammation and lipid signaling in the etiology of insulin resistance. Cell Metab 2012;15:635-645.

18 Wen H, Gris D, Lei Y, Jha S, Zhang L, Huang MT, Brickey WJ, Ting JP: Fatty acid-induced NLRP3-ASC inflammasome activation interferes with insulin signaling. Nat Immunol 2011;12:408-415.

19 Moschen AR, Kaser S, Tilg H: Non-alcoholic steatohepatitis: a microbiota-driven disease. Trends Endocrinol Metab 2013;24:537-545.

20 Sun Z, Lazar MA: Dissociating fatty liver and diabetes. Trends Endocrinol Metab 2013;24:4-12.

21 McPherson S, Hardy T, Henderson E, Burt AD, Day $\mathrm{CP}$, Anstee QM: Evidence of NAFLD progression from steatosis to fibrosing-steatohepatitis using paired biopsies: implications for prognosis and clinical management. J Hepatol 2015;62:1148-1155.

22 Tilg H, Diehl AM: Cytokines in alcoholic and nonalcoholic steatohepatitis. New Engl J Med 2000;343:14671476.

23 Moschen AR, Molnar C, Geiger S, Graziadei I, Ebenbichler CF, Weiss H, Kaser S, Kaser A, Tilg H: Antiinflammatory effects of excessive weight loss: potent suppression of adipose interleukin 6 and tumour necrosis factor alpha expression. Gut 2010;59:1259-1264.

24 Cai D, Yuan M, Frantz DF, Melendez PA, Hansen L, Lee J, Shoelson SE: Local and systemic insulin resistance resulting from hepatic activation of IKK-beta and NF-kappaB. Nat Med 2005;11:183-190.

-25 Arkan MC, Hevener AL, Greten FR, Maeda S, Li ZW, Long JM, Wynshaw-Boris A, Poli G, Olefsky J, Karin M: IKK-beta links inflammation to obesity-induced insulin resistance. Nat Med 2005;11:191-198.

26 Kiechl S, Wittmann J, Giaccari A, et al: Blockade of receptor activator of nuclear factor-kappaB (RANKL) signaling improves hepatic insulin resistance and prevents development of diabetes mellitus. Nat Med 2013; 19:358-363.

27 Scherer PE, Williams S, Fogliano M, Baldini G, Lodish HF: A novel serum protein similar to $\mathrm{C} 1 \mathrm{q}$, produced exclusively in adipocytes. J Biol Chem 1995;270: 26746-26749.

28 Ouchi N, Kihara S, Arita Y, Maeda K, Kuriyama H, Okamoto Y, Hotta K, Nishida M, Takahashi M, Nakamura T, Yamashita S, Funahashi T, Matsuzawa Y: Novel modulator for endothelial adhesion molecules: adipocyte-derived plasma protein adiponectin. Circulation 1999;100:2473-2476.

29 Yokota T, Oritani K, Takahashi I, Ishikawa J, Matsuyama A, Ouchi N, Kihara S, Funahashi T, Tenner AJ, Tomiyama Y, Matsuzawa Y: Adiponectin, a new member of the family of soluble defense collagens, negatively regulates the growth of myelomonocytic progenitors and the functions of macrophages. Blood 2000;96:1723-1732.

30 Maeda N, Shimomura I, Kishida K, Nishizawa H, Matsuda M, Nagaretani H, Furuyama N, Kondo H, Takahashi M, Arita Y, Komuro R, Ouchi N, Kihara S, Tochino Y, Okutomi K, Horie M, Takeda S, Aoyama T, Funahashi T, Matsuzawa Y: Diet-induced insulin resistance in mice lacking adiponectin/ACRP30. Nat Med 2002;8:731-737.

31 Wolf AM, Wolf D, Rumpold H, Enrich B, Tilg H: Adiponectin induces the anti-inflammatory cytokines IL-10 and IL-1RA in human leukocytes. Biochem Biophys Res Commun 2004;323:630-635.
32 Mandal P, Park PH, McMullen MR, Pratt BT, Nagy LE: The anti-inflammatory effects of adiponectin are mediated via a heme oxygenase-1-dependent pathway in rat Kupffer cells. Hepatology 2010;51:1420-1429.

33 Bruun JM, Lihn AS, Verdich C, Pedersen SB, Toubro S, Astrup A, Richelsen B: Regulation of adiponectin by adipose tissue-derived cytokines: in vivo and in vitro investigations in humans. Am J Physiol Endocrinol Metab 2003;285:E527-533.

34 Turer AT, Scherer PE: Adiponectin: mechanistic insights and clinical implications. Diabetologia 2012;55: 2319-2326.

35 Wolf AM, Wolf D, Avila MA, Moschen AR, Berasain C, Enrich B, Rumpold H, Tilg H: Up-regulation of the anti-inflammatory adipokine adiponectin in acute liver failure in mice. J Hepatol 2006;44:537-543.

36 Marchesini G, Brizi M, Morselli-Labate AM, Bianchi G, Bugianesi E, McCullough AJ, Forlani G, Melchionda N: Association of nonalcoholic fatty liver disease with insulin resistance. Am J Med 1999;107:450455.

37 Matthews DR, Hosker JP, Rudenski AS, Naylor BA, Treacher DF, Turner RC: Homeostasis model assessment: insulin resistance and beta-cell function from fasting plasma glucose and insulin concentrations in man. Diabetologia 1985;28:412-419.

38 Fraser A, Harris R, Sattar N, Ebrahim S, Davey Smith G, Lawlor DA: Alanine aminotransferase, gamma-glutamyltransferase, and incident diabetes: the British Women's Heart and Health Study and meta-analysis. Diabetes Care 2009;32:741-750.

39 Goessling W, Massaro JM, Vasan RS, D’Agostino RB Sr, Ellison RC, Fox CS: Aminotransferase levels and 20-year risk of metabolic syndrome, diabetes, and cardiovascular disease. Gastroenterology 2008;135:19351944, 1944.e1931.

40 Armstrong MJ, Adams LA, Canbay A, Syn WK: Extrahepatic complications of nonalcoholic fatty liver disease. Hepatology 2014;59:1174-1197.

41 Yki-Jarvinen H: Non-alcoholic fatty liver disease as a cause and a consequence of metabolic syndrome. Lancet Diabetes Endocrinol 2014;2:901-910.

42 Seppala-Lindroos A, Vehkavaara S, Hakkinen AM, Goto T, Westerbacka J, Sovijarvi A, Halavaara J, YkiJarvinen H: Fat accumulation in the liver is associated with defects in insulin suppression of glucose production and serum free fatty acids independent of obesity in normal men. J Clin Endocrinol Metab 2002;87: 3023-3028.

43 Pais R, Charlotte F, Fedchuk L, Bedossa P, Lebray P, Poynard T, Ratziu V: A systematic review of follow-up biopsies reveals disease progression in patients with non-alcoholic fatty liver. J Hepatol 2013;59:550-556.

44 Leite NC, Salles GF, Araujo AL, Villela-Nogueira CA, Cardoso CR: Prevalence and associated factors of nonalcoholic fatty liver disease in patients with type-2 diabetes mellitus. Liver Int 2009;29:113-119.

45 Targher G, Bertolini L, Padovani R, Rodella S, Tessari R, Zenari L, Day C, Arcaro G: Prevalence of nonalcoholic fatty liver disease and its association with cardiovascular disease among type 2 diabetic patients. Diabetes Care 2007;30:1212-1218.

46 Williams CD, Stengel J, Asike MI, Torres DM, Shaw J, Contreras M, Landt CL, Harrison SA: Prevalence of nonalcoholic fatty liver disease and nonalcoholic steatohepatitis among a largely middle-aged population utilizing ultrasound and liver biopsy: a prospective study. Gastroenterology 2011;140:124-131.
47 Doycheva I, Cui J, Nguyen P, Costa EA, Hooker J, Hofflich H, Bettencourt R, Brouha S, Sirlin CB, Loomba R: Non-invasive screening of diabetics in primary care for NAFLD and advanced fibrosis by MRI and MRE. Aliment Pharm Ther 2016;43:83-95

48 Abrams GA, Kunde SS, Lazenby AJ, Clements RH: Portal fibrosis and hepatic steatosis in morbidly obese subjects: a spectrum of nonalcoholic fatty liver disease. Hepatology 2004;40:475-483.

49 Hyysalo J, Mannisto VT, Zhou Y, et al: A populationbased study on the prevalence of NASH using scores validated against liver histology. J Hepatol 2014;60: 839-846.

50 Loomba R, Abraham M, Unalp A, Wilson L, Lavine J, Doo E, Bass NM: Association between diabetes, family history of diabetes, and risk of nonalcoholic steatohepatitis and fibrosis. Hepatology 2012;56:943-951.

51 Angulo P, Keach JC, Batts KP, Lindor KD: Independent predictors of liver fibrosis in patients with nonalcoholic steatohepatitis. Hepatology 1999;30:1356-1362.

52 Byrne CD, Targher G: NAFLD: a multisystem disease. J Hepatol 2015;62:S47-64.

53 Sanyal AJ, Chalasani N, Kowdley KV, McCullough A, Diehl AM, Bass NM, Neuschwander-Tetri BA, Lavine JE, Tonascia J, Unalp A, Van Natta M, Clark J, Brunt EM, Kleiner DE, Hoofnagle JH, Robuck PR: Pioglitazone, vitamin E, or placebo for nonalcoholic steatohepatitis. New Engl J Med 2010;362:1675-1685.

54 Ratziu V: Pharmacological agents for NASH. Nat Rev Gastroenterol Hepatol 2013;10:676-685.

55 Armstrong MJ, Gaunt P, Aithal GP, Barton D, Hull D, Parker R, Hazlehurst JM, Guo K, Abouda G, Aldersley MA, Stocken D, Gough SC, Tomlinson JW, Brown RM, Hubscher SG, Newsome PN: Liraglutide safety and efficacy in patients with non-alcoholic steatohepatitis (LEAN): a multicentre, double-blind, randomised, placebo-controlled phase 2 study. Lancet 2016;387: 679-690.

56 Thomas C, Pellicciari R, Pruzanski M, Auwerx J, Schoonjans K: Targeting bile-acid signalling for metabolic diseases. Nat Rev Drug Discov 2008;7:678-693.

57 Neuschwander-Tetri BA, Loomba R, Sanyal AJ, Lavine JE, Van Natta ML, Abdelmalek MF, Chalasani N, Dasarathy S, Diehl AM, Hameed B, Kowdley KV, McCullough A, Terrault N, Clark JM, Tonascia J, Brunt EM, Kleiner DE, Doo E: Farnesoid X nuclear receptor ligand obeticholic acid for non-cirrhotic, non-alcoholic steatohepatitis (FLINT): a multicentre, randomised, placebo-controlled trial. Lancet 2015;385: 956-965.

58 Ratziu V, Harrison SA, Francque S, Bedossa P, Lehert P, Serfaty L, Romero-Gomez M, Boursier J, Abdelmalek M, Caldwell S, Drenth J, Anstee QM, Hum D, Hanf R, Roudot A, Megnien S, Staels B, Sanyal A: Elafibranor, an agonist of the peroxisome proliferatoractivated receptor-alpha and -delta, induces resolution of nonalcoholic steatohepatitis without fibrosis worsening. Gastroenterology 2016;150:1147-1159.e1145. 